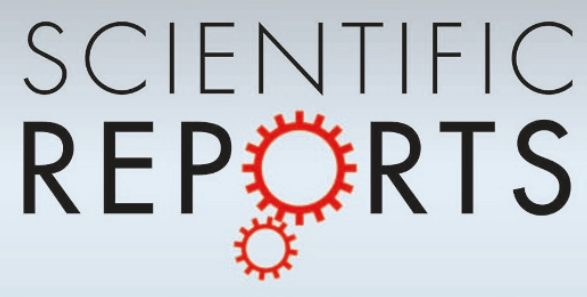

OPEN

SUBJECT AREAS:

ELECTRONIC PROPERTIES

AND MATERIALS

SURFACES, INTERFACES AND

THIN FILMS

QUANTUM MECHANICS

Received

17 July 2013

Accepted

8 November 2013

Published

3 December 2013

Correspondence and requests for materials should be addressed to B.Ö. (barbaros@nus. edu.sg)

* Current address: Department of Physics, India Institute of

Technology Madras, Chennai 600036 India.

\section{Multiple Virtual Tunneling of Dirac Fermions in Granular Graphene}

\author{
Alexandre Pachoud ${ }^{1,2,3}$, Manu Jaiswal ${ }^{1,3,4 *}$, Yu Wang ${ }^{3,4}$, Byung-Hee Hong ${ }^{5}$, Jong-Hyun Ahn ${ }^{6}$, \\ Kian Ping Loh ${ }^{3,4} \&$ Barbaros Özyilmaz ${ }^{1,2,3,7}$
}

'Department of Physics, National University of Singapore, Singapore 1 17542, Singapore, ${ }^{2}$ NUS Graduate School for Integrative Sciences and Engineering (NGS), Singapore 1 17456, Singapore, ${ }^{3}$ Graphene Research Center, National University of Singapore, Singapore 117542, Singapore, ${ }^{4}$ Department of Chemistry, National University of Singapore, Singapore 117543 , Singapore, ${ }^{5}$ Department of Chemistry, Seoul National University, Seoul 152-742, Korea, ${ }^{6}$ School of Electrical \& Electronic Engineering, Yonsei University, Seoul, 120-749, Korea, ${ }^{7}$ Nanocore, 4 Engineering Drive 3, National University of Singapore 117576, Singapore.

Graphene charge carriers behave as massless Dirac fermions, opening the exciting possibility to observe long-range virtual tunneling of electrons in a solid. In granular metals, electron hops arising from series of virtual transitions are predicted to yield observable currents at low-enough temperatures, but to date experimental evidence is lacking. We report on electron transport in granular graphene films self-assembled by hydrogenation of suspended graphene. While the log-conductance shows a characteristic $\mathrm{T}^{-1 / 2}$ temperature dependence, cooling the samples below $10 \mathrm{~K}$ drives a triple crossover: a slope break in log-conductance, simultaneous to a substantial increase in magneto-conductance and onset of large mesoscopic conductance fluctuations. These phenomena are signatures of virtual transitions of electrons between distant localized states, and conductance statistics reveal that the high crossover-temperature is due to the Dirac nature of granular graphene charge carriers.

hort-lived particles allowed by Heisenberg's uncertainty principle are called "virtual", but the effects they induce are very real. They lead to vacuum fluctuations and mediate fundamental forces ${ }^{1}$, explain the Lamb shift of atomic levels ${ }^{2}$, the Casimir effect ${ }^{3}$ and possible Hawking radiations ${ }^{4}$. Though unobservable, virtual particles are key ingredients of modern quantum electrodynamics. Considerable research efforts have thus been devoted to measuring the most direct consequences of their existence, such as virtual-to-real photon conversion during dynamic Casimir effect ${ }^{5}$. Quantum dot nanostructures are excellent test beds too, as electron tunneling through virtual states ${ }^{6-8}$ generates background currents observable below $\sim 100 \mathrm{mK}^{9}$. Interestingly, higher-order currents between distant localized states are predicted to arise from multiple transitions to virtual states in macroscopic granular metals ${ }^{10-12}$, but this phenomenon coined multiple elastic co-tunneling (MEC) has not been observed yet. From this perspective, graphene is a particularly promising material, in which virtual excitations of charges play a special role. They are theoretically predicted to induce Dirac fermions' jittery motion called zitterbewegung $^{13}$, and give a minimum conductivity to pristine graphene. In granular form, graphene dots' linear density of states (DOS $)^{14}$ means that high-energy virtual states should contribute significantly to elastic cotunneling currents despite shorter life-times, unlike ordinary granular metals (GM). This not only makes granular graphene the ideal platform for the first observation of MEC, but also provides a rare opportunity to measure long-range effects mediated by high-energy virtual states.

\section{Results}

Recently, chemical functionalization of graphen ${ }^{15}$ was reported to be a viable method to produce granular graphen ${ }^{16}$, due to the tendency of adatoms to form electrically insulating clusters. At high enough adatoms concentrations, such clusters merge into percolative pathways, effectively partitioning the graphene sheet into weakly-coupled graphene dot arrays, or GM. To conveniently fabricate graphene GMs, we exposed suspended CVD graphene sheets ${ }^{17}$ to hydrogen plasma ${ }^{18,19}$, thereby allowing adsorption of sufficiently high concentrations of hydrogen atoms on both sides of the graphene scaffold. We then stamped as-produced doubly-hydrogenated graphene (DHG) films on $90 \mathrm{~nm}-\mathrm{SiO}_{2}$ chips. $\mathrm{Au} / \mathrm{Cr}$ contacts in two- and four-probe geometries were then fabricated. Typical devices are shown in inset of figure 1(a). To observe the charge-neutrality point (CNP) at gate voltages $V_{g} \sim 0 \mathrm{~V}$, the samples were then vacuum-dried ${ }^{20}$ in-situ at $10^{-6}$ Torr for a day before cooling below $0^{\circ} \mathrm{C}$. We then measured electron transport in 8 devices from room temperature down to $2.4 \mathrm{~K}$. Figure 1(a) shows 

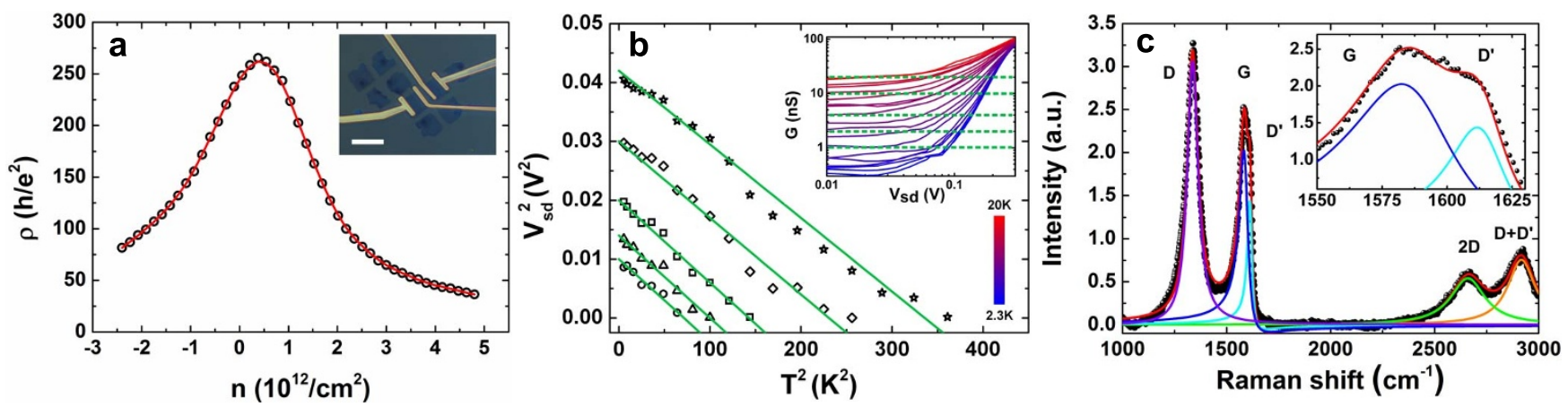

Figure $1 \mid$ (a) Sheet resistance against charge density n at room temperature (Device D0). Inset shows an optical picture of typical devices. Scale bar: $10 \mu \mathrm{m}$. (b) Constant-conductance $V_{s d}{ }^{2}$ vs $T^{2}$ domains extracted from conductance $G$ against $V_{s d}$ curves at $2.3 \mathrm{~K}$ and all temperatures between $3 \mathrm{~K}$ and $20 \mathrm{~K}$ in steps of $1 \mathrm{~K}$ (inset). The solid lines of the main figure are linear fits of slope $\sim 1.4 \times 10^{-4} \mathrm{~V}^{2} \mathrm{~K}^{-2}$ to $G=1 \mathrm{nS}$ (circles), $G=2 \mathrm{nS}$ (triangles), $G=$ $4 \mathrm{nS}$ (squares), $G=10 \mathrm{nS}$ (diamonds) and $G=20 \mathrm{nS}$ (stars) domains. These domains correspond to traces represented as green dashed lines in inset. (c) Raman spectrum (device D1). The data (black) are fitted by a five-peak line-shape (red), sum of Breit-Wigner-Fano peaks: violet (D), blue $(G)$, cyan $\left(D^{\prime}\right)$, green $(2 D)$ and orange $(D+G)$. The inset is a zoom on the $G$ and $D^{\prime}$ peaks.

the room-temperature resistance $R$ in units of resistance quantum $h / e^{2}$, against charge-carrier density $n$ for a typical sample (D0). The $R(n)$ curve has a characteristic graphene-like shape, but is broad and $R(n) \gg 1$ throughout the whole range of measured densities, indicating strong localization.

Next, we measure the conductance $G$ for different bias voltages $V_{s d}$ and temperatures $T$ to extract the typical localization length $\xi$ of our samples. The inset of figure 1(b) shows $G$ against $V_{s d}$ at different temperatures $T$ between $2.3 \mathrm{~K}$ and $20 \mathrm{~K}$ for device D1. For consistency, all subsequent data shown in main text correspond to the same device D1. Data taken for other devices are reported in Supplementary Material, section A. We observe that above $V_{s d} \sim$ $0.1 \mathrm{~V}, G$ increases with $V_{s d}$ while below, $G$ is bias-independent, indicating an Ohmic behavior. Crucially, $G\left(V_{s d}, T\right)$ data can be used to extract $\xi$ without assuming any particular transport mechanism. Since an electron hopping against the source-drain electric field $E$ $=V_{s d} / L$ over a distance $d$ increases its energy by an amount $e E d$, it was shown that charge carriers experience an effective temperature $T_{\text {eff }}=\sqrt{T^{2}+\left(\alpha e \xi V_{s d} / k_{B} L\right)^{2}}$ where $L=4 \mu \mathrm{m}$ is the channel length, and $\alpha \approx 0.67$ is a constant ${ }^{21}$. Importantly, $T_{\text {eff }}$ uniquely determines $G$, which implies that constant-conductance domains of $\left(V_{s d}{ }^{2}, T^{2}\right)$ space are straight lines of slope $-\left(\alpha e \xi / k_{B} L\right)^{2}$. Figure $1(\mathrm{~b})$ shows such domains extracted from $G\left(V_{s d}, T\right)$ data at $1,2,4,10$ and $20 \mathrm{nS}$. As expected, they are well-fitted by straight lines of slope $\sim-1.4 \times$ $10^{-4} \mathrm{~V}^{2} \mathrm{~K}^{-2}$, giving $\xi \approx 45 \mathrm{~nm}$.

We now show that our DHG samples have a GM structure by complementing our electric transport measurements with Raman spectroscopy data, presented in figure $1(\mathrm{c})$. The spectrum shown in figure $1(\mathrm{c})$ exhibits a prominent $\mathrm{D}$ peak as well as broad $2 \mathrm{D}$ and $\mathrm{D}+\mathrm{D}^{\prime}$ peaks. The $\mathrm{D}$ peak partially overlaps with the $\mathrm{G}$ peak. The $\mathrm{D}^{\prime}$ peak at $1614 \mathrm{~cm}^{-1}$ almost completely merges with the $\mathrm{G}$ peak at $1588 \mathrm{~cm}^{-1}$ but can still be resolved as shown by the inset of figure $1(\mathrm{c})$. These are characteristics of strongly $\mathrm{sp}^{3}$-hybridized graphene $\mathrm{e}^{22}$. The D-peak of $\mathrm{sp}^{3}$-hybridized graphene probes the distribution of $\mathrm{sp}^{3}$-bonds on the graphene lattice ${ }^{23}$. More precisely, the ratio $I_{D} / I_{G}$ of integrated intensities of the $\mathrm{D}$ and $\mathrm{G}$ peaks is related to the typical adatom cluster size $r_{s}$ and the mean distance $L_{D}$ between nearest cluster centers by the following formula ${ }^{24,25}$ :

$\frac{I_{D}}{I_{G}}=F\left(\mathrm{r}_{s}, \mathrm{~L}_{D}\right)=\mathrm{C}_{A} \frac{r_{A}^{2}-r_{S}^{2}}{r_{A}^{2}-2 r_{S}^{2}}\left[e^{-\frac{\pi r_{S}^{2}}{L_{D}^{2}}}-e^{-\frac{\pi\left(r_{A}^{2}-r_{S}^{2}\right)}{L_{D}^{2}}}\right]+C_{S}\left[1-e^{-\frac{\pi r_{S}^{2}}{L_{D}^{2}}}\right](1)$

where $C_{A}$ and $C_{S}$ are constants, $r_{A}=r_{S}+\delta$, and $\delta$ is the average distance laser-excited electrons travel before recombining with holes. Experimentally, it was shown that $C_{A} \sim 4, C_{S} \sim 0.9$ and $\delta \sim 2 \mathrm{~nm}$ for a laser of $2.4 \mathrm{eV}^{26,27}$. By fitting Fano line-shapes ${ }^{28}$ to the Raman spectrum peaks, we calculate the peaks integrated intensities and $I_{D} / I_{G} \sim 1.8$. This value is clearly inconsistent with a random distribution of isolated adatoms, which would yield $L_{D} \sim \delta \ll \xi$. Conversely, hydrogen adatoms form clusters of typical radius $r_{s}^{29}$. Simple geometric considerations discussed in section B of Supplementary Material impose that $L_{D}, r_{s}$ and $\xi$ typically follow the relation $\sqrt{2} L_{D} \approx 2 r_{S}+\xi$. Since $\xi \approx 45 \mathrm{~nm}$, we find $r_{s} \sim 30 \mathrm{~nm}$ by solving $F\left(r_{s}, L_{D}\right) \approx 1.8$, leading to $L_{D} \approx 2 r_{s}$. In other words, clusters tend to merge, isolating graphene dots of size $\xi$, and our DHG samples have a GM structure. This agrees with previous studies on graphene quantum dots of size $\sim \xi^{30}$ yielding comparable Raman spectra due to edge scattering ${ }^{26,31}$.

Next, we focus on identifying the dominant charge transport mechanisms by analyzing the temperature-dependence of the conductance $G(T)$. More precisely, one expects $G$ to follow a $G=$ $G_{0} \exp \left(-\left(T_{0} / T\right)^{\gamma}\right)$ law characteristic of hopping transport, where $\gamma$ and $T_{0}$ depend on the exact hopping mechanism ${ }^{32}$. We thus measured $G$ around CNP for different $T$ between $2.4 \mathrm{~K}$ and $300 \mathrm{~K} . G(T)$ is systematically measured in the low-bias Ohmic regime, where both electric-field-driven electron hopping and Joule heating are negligible. As shown in section $C$ of Supplementary Material, we observed a reduced activation energy ${ }^{33} \beta=d \ln G / d \ln T$ linear in $\ln G$ with slope $\sim-1 / 2$ both for $\ln G<-21.5$ and $\ln G>-19$. Therefore, we plotted $\mathrm{G}$ against $T^{-1 / 2}$ in figure 2(a). Strikingly, $\ln G$ $\propto T^{-1 / 2}$ between $300 \mathrm{~K}$ and $\sim 12 \mathrm{~K}$, and between $\sim 8 \mathrm{~K}$ and $2.4 \mathrm{~K}$, but with a much smaller slope. A distinct slope break is thus identified around $\mathrm{T}_{\text {cross }}=10 \mathrm{~K}$. Graphene being atomically thin, this phenomenon certainly does not reflect a decrease in effective sample dimensionality from three to two dimensions ${ }^{34}$. Besides, our measured samples have a channel width $\mathrm{W} \approx 6-7 \mu \mathrm{m}$ systematically larger than the length $L \leq 5 \mu \mathrm{m}$ to avoid any possible 2D to $1 \mathrm{D}$ crossover upon lowering the temperature. A $G_{0} \exp \left(-\left(T_{0} / T\right)^{\gamma}\right)$ fit both above and below $T_{\text {cross }}$ respectively gives $\gamma=0.56 \pm 0.04$ and $\gamma=0.495 \pm 0.05$. In these regimes, $G(T)$ is thus neither of the Arrhenius type $(\gamma=1)$ nor of the Mott's 2D variable-range hopping (VRH) type $(\gamma=1 / 3)$. However, $\gamma=1 / 2$ suggests an EfrosShklovskii (ES) VRH behavior, the 1D Mott VRH being excluded due to the geometry of our devices. Such behavior contrasts with the result of several earlier studies, in particular ${ }^{18}$, performed on strongly localized hydrogenated graphene samples, where the conduction is attributed to 2D Mott VRH. However, these results were obtained for less hydrogenated graphene samples, fabricated by exposing a single graphene face to hydrogen plasma. Conversely, a $\gamma=1 / 2$ behavior was already reported in heavily oxidized graphene films ${ }^{16}$ with a GM structure. However, the presence of both a gamma-1/2 behavior and 

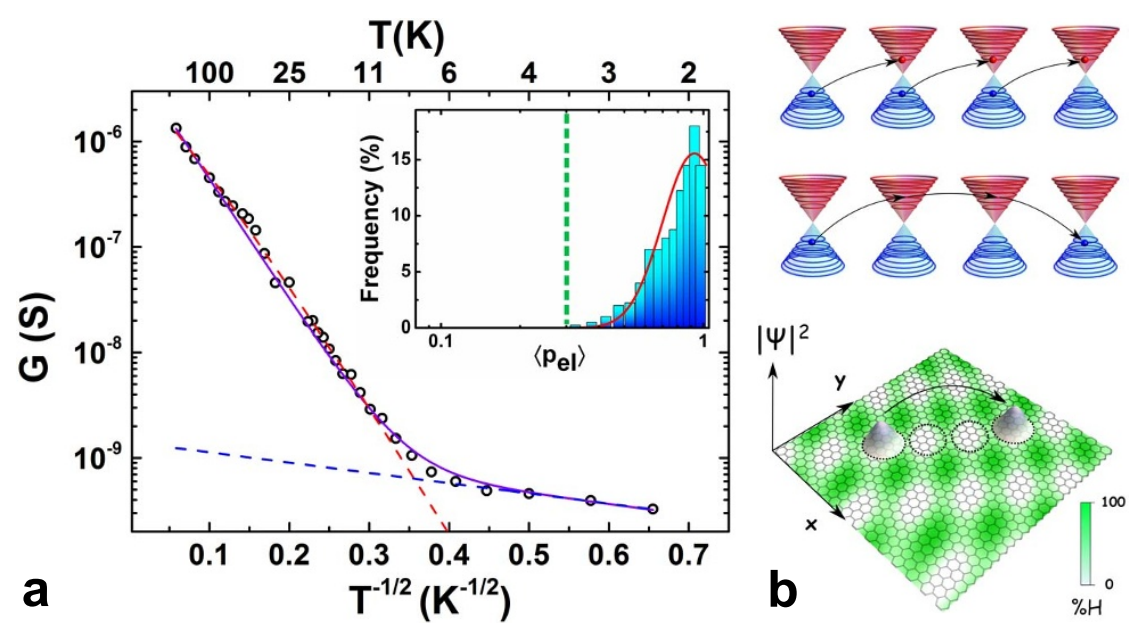

Figure $2 \mid$ (a) Low-bias $G$ vs $T^{-1 / 2}$ at $V_{g}=1.5 \mathrm{~V}$. The violet line is a fit accounting for inelastic and elastic co-tunneling mechanisms as described in main text. The blue (resp. red) dashed line corresponds to the best power law fit for $\ln G$ vs $T^{-1 / 2}$ below $6 \mathrm{~K}$ (resp. above $10 \mathrm{~K}$ ). Inset shows a histogram for $p_{e l}$ extracted from slopes of $\ln G(T)$ vs $T^{-1 / 2}$ at different $V_{g}$, shown in Supplementary Material, section D. The red solid line is a guide to the eyes and the dashed green line is the lower-bound for $p_{e l}$ calculated in Supplementary Material, section E. (b) Cartoon representation of hydrogenated graphene sheets (bottom), MEC (centre) and MIC (top). Hydrogen clusters partition hydrogenated graphene into disconnected metallic graphene dots. Hydrogen concentration is encoded by shades of green, from white (hydrogen-poor) to green (hydrogen-rich). Hopping from initial to final localized state (grey) occurs by two possible mechanisms described in main text: MIC (top) and MEC (centre). Cones represent graphene's energy spectrum within individual dots circled by dashed lines in bottom panel. Discrete energy levels due to confinement are marked by red and blue circles. Red (blue) balls represent electrons (holes). Arrows correspond to transitions due to absorption of phonons (top) or virtual transitions (bottom).

a slope break as observed in figure 2(a) around $10 \mathrm{~K}$ has never been observed before, to the best of our knowledge. While a crossover between two $\gamma=1 / 2$ regimes with different slopes is not expected in standard VRH theories ${ }^{32}$, it is predicted for granular systems ${ }^{10}$, each regime reflecting a distinct transport mechanism illustrated in figure 2(b): multiple inelastic co-tunneling (MIC) at high temperatures and multiple elastic co-tunneling (MEC) at low temperatures. During MIC, multiple electrons simultaneously tunnel from the Fermi sea of a grain to an excited state of a neighboring grain, along a string of grains ultimately left in an excited state, thus requiring a finite temperature or electric field. During MEC, charge carriers tunnel between distant grains by transiting to intermediate virtual states, without absorbing or emitting phonons. To verify that our $\mathrm{G}(\mathrm{T})$ data result from a MIC-to-MEC crossover, we fit a function of the form $G(T)=G_{M I C}(T)+G_{M E C}(T)$, where $G_{M I C}(T)=$ $G_{i n} \exp \left(-\sqrt{T_{i n} / T}\right)$ and $G_{M E C}(T)=G_{e l} \exp \left(-\sqrt{T_{e l} / T}\right)$ are MIC and MEC conductance terms. The best fit is obtained for $G_{i n} \approx$ $6 \mu \mathrm{S}, T_{\text {in }} \approx 700 \mathrm{~K}, G_{e l} \approx 1.5 \mathrm{nS}$, and $T_{e l} \approx 6 \mathrm{~K}$. We now show that these values match theoretical expectations for co-tunneling in granular graphene. We start with $G_{i n}$, which is the total conductance in the $T \gg T_{i n}$ limit and is related to the nearest-grain tunneling conductance $\mathrm{g}$ by $g \sim L G_{i n} / W$, where $W \approx 7 \mu \mathrm{m}$ is the sample width. This leads to $g \sim 0.1 \ll 1$ in units of $e^{2} / h$, which is a characteristic of insulating GM. The inter-dot tunneling conductance $g$ should also be compared to the intra-dot conductance $g_{d o t}{ }^{35}$. Since before hydrogenation, our graphene samples have a typical mobility $\mu \approx$ $5000 \mathrm{~cm}^{2} / \mathrm{V}$.s, the intra-dot mean-free path is $l=\mathrm{A} \sqrt{n}$ where $\mathrm{A}=h \mu / 2 \sqrt{\pi} e \approx 6 \times 10^{-12} \mathrm{~cm}^{2}$. A graphene grain of size $\sim \xi$ with at least one Dirac fermion, corresponding to an areal density of $\sim 5 \times$ $10^{10} / \mathrm{cm}^{2}$, thus has $l>13 \mathrm{~nm}$ and a Thouless energy ${ }^{36} E_{T h}=2 \hbar v_{F} l / \xi^{2}$ larger than $\sim 10 \mathrm{meV}$. Therefore, $E_{T h}$ typically exceeds the dots mean energy level spacing ${ }^{37} \Delta \leq \hbar v_{F} / \xi \sim 10 \mathrm{meV}$, so that $g_{d o t} \approx 2 e^{2} E_{T h} / h \Delta$ is at least a few $e^{2} / h . g_{\text {dot }} \gg g$ is thus satisfied throughout the experimentally relevant range of densities. This is again perfectly consistent with an insulating granular metal behavior, and contrasts sharply with the case of homogeneously disordered systems ${ }^{10}$, where $g_{\text {dot }} \sim g$. Next, we notice that $T_{\text {in }} \approx 700 \mathrm{~K}$ is significantly larger than the charging energy of a grain $T_{c}=e^{2} / 4 \pi \varepsilon \varepsilon_{0} \xi \approx 100 \mathrm{~K}$, where $\varepsilon \approx 3.5$ is the dielectric constant of the inter-granular medium ${ }^{16}$. This agrees with MIC theory ${ }^{10}$ which predicts $T_{i n}=\chi_{i n} T_{c}$, where $\chi_{\text {in }}$ is a logarithmically $T$-dependent coefficient. As $T \ll T_{c}, \chi_{\text {in }}=-4 \ln p_{\text {in }}$ where $p_{i n} \approx 16 \pi g\left(T / T_{c}\right)^{2}$ is the rate of inelastic tunneling between two neighboring grains ${ }^{12}$, giving $\chi_{\text {in }} \approx 8$ at $T=10-20 \mathrm{~K}$, and hence $T_{\text {in, theory }} \approx 800 \mathrm{~K}$, close to the experimental value. We finally focus on $T_{e l}$, whose value extracted from figure $2(\mathrm{a})$ is one order of magnitude smaller than $T_{c}$. This remains true at all $V_{g}$ despite fluctuations, and $T_{e l}$ averages to $\theta_{e l} \approx 10 \mathrm{~K}$. More details on the statistics of $T_{e l}$ are reported in Supplementary Material, section D. Theoretically, $2 T_{e l}=-T_{c} \ln p$, where $\mathrm{p}$ is the probability of virtual transition to a neighboring grain ${ }^{10,11}$. For conventional two-dimensional GMs, the DOS in each grain is constant and the level spacing is small compared to the charging energy, leading to ${ }^{10} p \propto g \Delta / E_{c} \ll g \ll 1$ and $T_{e l}$ of the order of few $T_{c}$. Therefore, Schrödinger fermions cannot account for the observed $T_{e l} \ll T_{c}$ behavior. Unlike MIC for which only states within $k_{B} T_{\text {eff }}$ around the Fermi level contribute to $G$, virtual transitions to high-energy states contribute to the MEC conductance and the band structure plays a key role. In the Dirac fermions case, the short life-time $\hbar / \mathrm{E}$ of high-energy fermions is compensated by a DOS which increases linearly with energy $E$, making the contribution of high-energy virtual states significant. It remains true for chaotic dots with edges of random shape ${ }^{14,37}$. Following refs. 11 and 38, we calculated in Supplementary Material (section E) a lowerbound for the virtual transition probability of graphene's Dirac Fermions: $p_{e l} \geq g\left(\ln \left(\Gamma / T_{c}\right)-1\right) / 2 \approx 0.3$, where $\Gamma$ is the energy bandwidth. This is in accord with experimental statistics on $p_{e l}$ shown in inset of figure 2 (a) and implies that $p_{i n} \sim p_{e l}$ just above $T_{\text {cross }}$, between $20 \mathrm{~K}$ and $40 \mathrm{~K}$, thus providing strong evidence for MIC-to-MEC crossover.

A powerful way to gain further insight is to analyze the variations of $G$ with $V_{g}$ and $V_{s d}$, as each transport mechanism leaves its own mesoscopic fluctuations footprints ${ }^{38-41}$. Figure 3(a) shows $G$ measured at $3 \mathrm{~K}$ for $-7.5 \mathrm{~V} \leq V_{g} \leq 7.5 \mathrm{~V}$ and $0 \leq V_{s d} \leq 200 \mathrm{mV}$ and exhibits vertical stripes of width $\Delta V_{g} \sim 200 \mathrm{mV}$, corresponding to peaks and valleys in conductance reproduced at all measured $V_{s d}$. This is highlighted by figure $3(\mathrm{~b})$, which shows four different traces 

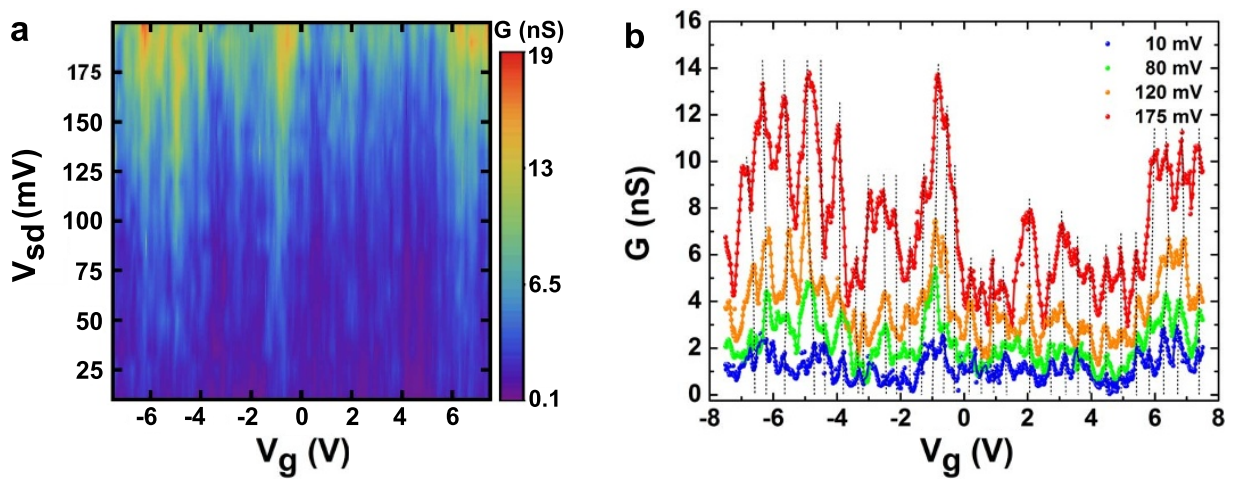

Figure $3 \mid$ (a) Conductance as a function of $V_{g}$ and $V_{s d}$ at $3 \mathrm{~K}$. The linear color scale corresponds to the measured conductance in nS. (b) Conductance against $V_{g}$ traces at $3 \mathrm{~K}$ extracted from fig. 3(a): at $V_{s d}=10 \mathrm{mV}$ (blue), $V_{s d}=80 \mathrm{mV}$ (green), $V_{s d}=120 \mathrm{mV}$ (orange), and $V_{s d}=175 \mathrm{mV}$ (red). The vertical black dashed curves are guides to the eyes highlighting the reproducibility of the peaks in conductance across different voltage biases.

extracted from figure $3(\mathrm{a})$ at $V_{s d}=10 \mathrm{mV}, 80 \mathrm{mV}, 120 \mathrm{mV}$ and $175 \mathrm{mV}$. While overall the conductance increases with $V_{s d}$, the different fixed- $V_{s d} G\left(V_{g}\right)$ curves show reproducible peaks and valleys. To analyze these fluctuations quantitatively, we systematically extracted the standard deviation of $\log$-conductance $\sigma_{\ln G}$ at fixed source-drain bias from figure 3(a) and three other data sets shown in Supplementary Material, section F. Figure 4 shows $\sigma_{\ln G}$ plotted against $T_{\text {eff }}$ from $3 \mathrm{~K}$ up to $80 \mathrm{~K}$, in double-log scale. Two different regimes can be clearly distinguished. Below $T_{\text {eff }}=$ $10 \mathrm{~K}, \sigma_{\ln G}$ is weakly temperature-dependent while above $10 \mathrm{~K}$, $\sigma_{\ln G}$ decreases rapidly with $T_{\text {eff. }}$ Quite remarkably, these two regimes coincide with the two distinct $G(T)$ regimes observed in figure 2(a). We start by analyzing the high- $T_{\text {eff }}$ regime, where $\sigma_{\ln G}$ is very well described by the power-law $\sigma_{\ln G} \propto T_{\text {eff }}^{-2.1}$. This behavior is clearly incompatible with Mott or ES VRH as such phenomena would lead to $\sigma_{\ln G} \propto T_{\text {eff }}^{-a}$ with $a<1^{41}$. We now compare the observed fluctuations above $10 \mathrm{~K}$ to expected MIC-induced fluctuations. From a hopping percolation viewpoint ${ }^{42}$, the fluctuations in log-conductance $\sigma_{\mathrm{Ln} G}$ are related to the standard deviation $s$ of log-conductance at the scale of a hopping distance $r_{\text {hop }}$, by $\sigma_{\ln G}=\kappa^{v} / l$ where $\kappa=2 \sqrt{2} s, l=L / r_{\text {hop }}$ and $v \approx 4 / 3$ is the critical exponent in two dimensions. Since MIC is a fundamentally phase-incoherent process involving a different charge carrier for each intermediate transition (see figure 2(b)), MIC-induced fluctuations in conductance do not originate from quantum interferences between electron wave functions. It must rather arise from fluctuations in inter-dot conductances accompanying changes in percolation network as gate voltage is tuned. From this perspective, we find - based on probabilistic arguments developed in Supplementary Material, section G1 and G3 - that $s \propto \sqrt{N_{\text {hop }} / N_{\text {th }}}$ where ${ }^{10} N_{\text {hop }}=r_{\text {hop }} / \xi \propto \sqrt{T_{\text {in }} / T_{\text {eff }}}$ and $\mathrm{N}_{\text {th }}$ is the number of energy levels accessible in a neighboring grain by emitting or absorbing a phonon. For a 2D GM with parabolic-band grains, $N_{t h} \sim k_{B} T_{\text {eff }} / \Delta$ and hence $s \propto \sqrt{\Delta / k_{B} T_{\text {eff }}}\left(T_{\text {in }} /\right.$ $\left.T_{\text {eff }}\right)^{1 / 4}$. This corresponds to $\sigma_{\ln G} \propto T^{-1.5}$, which does not satisfactorily fit the data. However, in graphene grains, energy levels are not evenly spaced. The $\mathrm{n}^{\text {th }}$ energy level from neutrality point has an expected value of energy $E_{n} \propto \Delta_{0} \sqrt{n}$ where $\Delta_{0}=\hbar v_{F} / \xi$. Therefore, $N_{t h} \propto\left(k_{B} T_{\text {eff }} / \Delta_{0}\right)^{2}$ and $s \propto\left(\Delta_{0} / k_{B} T_{\text {eff }}\right)\left(T_{\text {in }} / T_{\text {eff }}\right)^{1 / 4} \propto T_{\text {eff }}^{-1.25}$ (see Supplementary Material, section G2). This yields $\sigma_{\ln G} \propto T_{\text {eff }}^{-2.16}$, which agrees very well with the data. In other words, the unusually strong $T_{\text {eff }}$-dependence of $\sigma_{\ln G}$ above $10 \mathrm{~K}$ can only be understood in terms of inelastic co-tunneling of Dirac fermions.

We now discuss the sub- $T_{\text {cross }}$ regime, where $\sigma_{\ln G} \sim 0.5$ is weakly $T$-dependent, clearly ruling out MIC as dominant transport mechanism below $10 \mathrm{~K}$. This is perfectly consistent with figure 2(a) which indicates a crossover to MEC below $10 \mathrm{~K}$. Since MEC is a phase-preserving process, it is tempting to assign this behavior to quantum interferences between distinct phase-coherent charge-carrier paths ${ }^{43,44}$, a phenomenon known to produce almost $T_{\text {eff }}$-independent conductance fluctuations of large magnitude. This view is supported by magneto-transport experiments carried out at $2.4 \mathrm{~K}$ for $-500 \mathrm{mV} \leq V_{s d} \leq 500 \mathrm{mV}$. The inset of figure 4 shows the relative magneto-conductance $M C=G(8 \mathrm{~T}) / G(0 \mathrm{~T})$ against $T_{\text {eff }}$ from $2.4 \mathrm{~K}$ up to $40 \mathrm{~K} . M C$ is almost constant and close to 1 above $T_{\text {cross }}$ where MIC dominates, reflecting its phase-incoherent nature, whereas $M C$ rapidly increases to $\sim 2$ below $T_{\text {cross }}$, a manifestation of quantum interferences ${ }^{11,44,45}$ attributable to MEC. Moreover, figure 4 shows that the sub- $T_{\text {cross }}$ data are well-described by $\sigma_{\ln G} \propto T_{\text {eff }}^{-\eta}$ with $\eta \sim$ 0.16 or less, suggesting conduction is limited by a strongly resistive portion of the granular graphene film of size $\sim r_{\text {hop }}$, or "bottleneck".

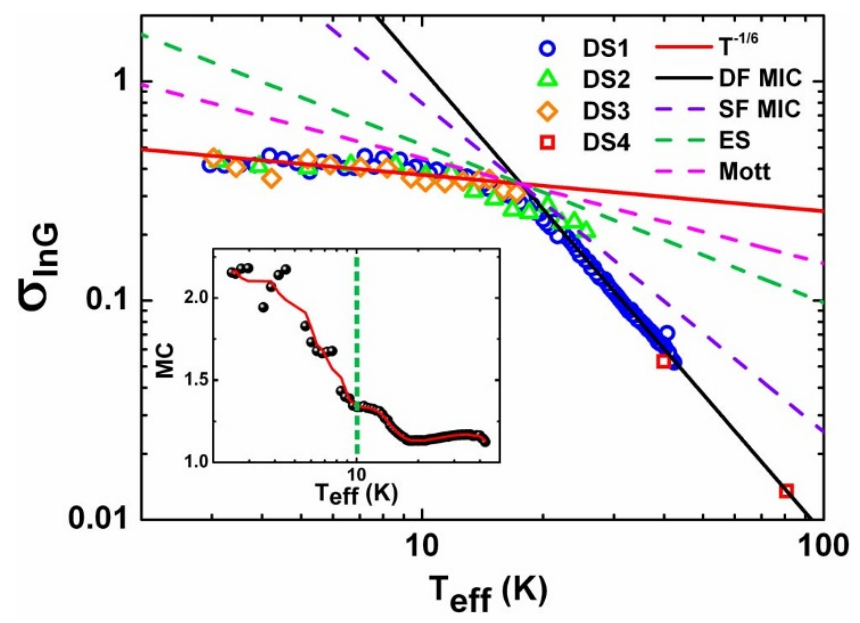

Figure $4 \mid$ Standard deviation $\sigma_{\ln G}$ of the $\log$-conductance as a function of $T_{\text {eff }}$. Data points were extracted from 4 distinct data sets - DS1, DS2, DS3, DS4, presented in Supplementary Material, section F - measured in sample D1. DS3 corresponds to fig. 3(a). Blue circles (DS1) were extracted from $G$ vs $V_{s d}$ curves taken at $3 \mathrm{~K}$ for different $V_{g}$. Green triangles (DS2) and orange diamonds (DS3) were extracted from $G$ vs $V_{g}$, $V_{s d}$ plots. Red squares (DS4) correspond to $G$ vs $V_{g}$ curves taken at $6 \mathrm{~K}$ and fixed source-drain current. Dashed and solid lines correspond to theoretically predicted power-laws for different transport mechanisms: Dirac fermions (DF) MIC (black), Schrödinger fermions (SF) MIC (violet), ES (green) and Mott $\mathrm{VRH}$ (pink), bottleneck-limited MEC (red). Inset shows the high-field $M C$ at $V_{g}=0 \mathrm{~V}$ between $2 \mathrm{~K}$ and $40 \mathrm{~K}$. Data points are shown in black. The solid red line is a guide to the eyes and the dashed green line indicates $T_{\text {cross }}$. 
Such a situation systematically occurs in granular media at sufficiently low $T_{\text {eff }}$ when $\sigma_{\text {LnG }}$ approaches unity ${ }^{41,46}$, which is the case around $T_{\text {cross. }}$. Since quantum interferences within a bottleneck must give the main contribution to $\sigma_{\ln G}$, the observed $\sigma_{\ln G} \propto T_{\text {eff }}^{-\eta}$-behavior plausibly approximates the $\sigma_{\ln G}=\left(A T_{\text {eff }}^{-1 / 3}+B\right)^{1 / 2}$ law for systems of size close to the phase-coherence length ${ }^{47-49}$. In summary, both the significant increase in magneto-conductance and weak temperature-dependence of $\sigma_{\ln G}$ below $10 \mathrm{~K}$ indicate the appearance of quantum interferences. The presence of such strong interferences only below $T_{\text {cross }}$ is consistent with the existence of a crossover from phase-incoherent MIC above $10 \mathrm{~K}$ to MEC below $10 \mathrm{~K}$.

\section{Discussion}

Our results show that doubly-hydrogenated graphene is not amorphous but has a granular structure instead. This finding is consistent with the tendency of hydrogen atoms adsorbed on graphene to phase-separate ${ }^{50}$ and form electrically insulating clusters. This observation is particularly interesting as graphane phases - or fully hydrogenated graphene regions - are predicted to be high-temperature superconductors ${ }^{51}$. From this perspective, hydrogenation of suspended graphene sheets appears as a viable route towards the synthesis of novel granular superconductors ${ }^{10,52}$. The granularity of our doubly-hydrogenated graphene samples is reflected by the presence of Dirac fermions in this material, which can exist in graphene grains or dots, but not in amorphous media. Both the large crossover temperature from inelastic to elastic co-tunneling and the temperature dependence of the log-conductance fluctuations with gate voltage are signatures of the presence of Dirac fermions in our samples.

In conclusion, we observed multiple elastic co-tunneling for the first time in a granular metal. In our granular graphene samples, both multiple inelastic and elastic co-tunneling mechanisms showed signatures of Dirac fermions. The presence of large high-order elastic co-tunneling currents in granular graphene establishes granular Dirac materials as ideal platforms for the study of vacuum fluctuations and quantum noise $\mathrm{e}^{53}$.

\section{Methods}

Device fabrication. CVD graphene was grown on copper foil using the technique described in ref. 17. The copper foil was etched away using an ammonium persulfate solution and transferred on a Quantifoil S7/2 square mesh holey carbon TEM grids, following a polymer-free transfer technique similar to ref. 54. Our freestanding graphene sheets were subsequently exposed to hydrogen plasma following the technique used in ref. 19. The resulting samples were deposited on $90 \mathrm{~nm}-\mathrm{SiO}_{2}$ chips immediately after plasma exposure, by gently stamping the TEM-grid-supported graphene sheet onto the substrate. This procedure generally left a large number of $7 \times$ $7 \mu^{2}$ square-shaped hydrogenated graphene flakes on $\mathrm{SiO}_{2}$, corresponding to the TEM grid mesh geometry. Devices were then fabricated by standard electron-beam lithography technique followed by thermal evaporation of $\mathrm{Au} / \mathrm{Cr}$ electrodes.

Electron transport measurement. After fabrication, samples were loaded in a variable temperature insert coupled to a $9 \mathrm{~T}$ superconducting magnet. A pressure of $\sim 10^{-6}$ Torr was maintained during the experiments. Electron transport measurements were carried out with a Keithley 6517B Electrometer/High-Resistance Meter. To eliminate possible DC noise, we used the following procedure: for each bias $V_{s d}$, the source-drain current $I_{s d}$ was measured 10 times at $+V_{s d}$ within $\sim 1 \mathrm{~s}$, then 10 times at $-V_{s d}$ within $\sim 1 \mathrm{~s}$. The resulting noise-filtered current was then systematically calculated as $\left(\left\langle I_{s d}\left(+V_{s d}\right)\right\rangle-\left\langle I_{s d}\left(-V_{s d}\right)\right\rangle\right) / 2$, where \langle\rangle corresponds to the 10-point average. $V_{s d}$ was sourced by Keithley $6517 \mathrm{~B}$ or Keithley 6430 . Keithley 6430 was systematically used to source the gate voltage. When needed, a fixed $I_{s d}$ was sourced with a Keithley 6221, while $V_{s d}$ was measured with Keithley 6517 B.

1. Peskin, M. E. \& Schroeder, D. V. An Introduction to Quantum Field Theory. (Addison-Wesley Publishing Company, Reading, 1995).

2. Scully, M. O. \& Zubairy, M. S. Quantum Optics. (Cambridge University Press, Cambridge, 1997).

3. Rodriguez, A. W., Capasso, F. \& Johnson, S. G. The Casimir effect in microstructured geometries. Nature Photon. 5, 211-221 (2011).

4. Hawking, S. W. Black hole explosions? Nature 248, 30-31 (1974).

5. Wilson, C. M. et al. Observation of the dynamical Casimir effect in a superconducting circuit. Nature 479, 376-379 (2011).
6. Averin, D. \& Nazarov, Y. Virtual electron diffusion during quantum tunneling of the electric charge. Phys. Rev. Lett. 65, 2446-2449 (1990).

7. Fujisawa, T. Spontaneous Emission Spectrum in Double Quantum Dot Devices. Science 282, 932-935 (1998).

8. De Franceschi, S. et al. Electron Cotunneling in a Semiconductor Quantum Dot. Phys. Rev. Lett. 86, 878-881 (2001).

9. Gustavsson, S. et al. Detecting single-electron tunneling involving virtual processes in real time. Phys. Rev. B 78, 155309 (2008).

10. Beloborodov, I., Lopatin, A., Vinokur, V. \& Efetov, K. Granular electronic systems. Rev. Mod. Phys. 79, 469-518 (2007).

11. Feigel'man, M. V. \& Ioselevich, A. S. Variable range cotunneling and conductivity of a granular metal. JETP Lett. 81, 341-347 (2005).

12. Beloborodov, I., Lopatin, A. \& Vinokur, V. Coulomb effects and hopping transport in granular metals. Phys. Rev. B 72, 125121 (2005).

13. Katsnelson, M. I. Zitterbewegung, chirality, and minimal conductivity in graphene. EPJ B 51, 157-160 (2006)

14. Berry, M. V. \& Mondragon, R. J. Neutrino Billiards: Time-Reversal SymmetryBreaking Without Magnetic Fields. Proc. R. Soc. A 412, 53-74 (1987).

15. Balakrishnan, J., Koon, G. K. W., Jaiswal, M., Castro Neto, A. H. \& Özyilmaz, B. B. Colossal enhancement of spin-orbit coupling in weakly hydrogenated graphene. Nature Phys. 9, 284-287 (2013).

16. Joung, D., Zhai, L. \& Khondaker, S. I. Coulomb blockade and hopping conduction in graphene quantum dots array. Phys. Rev. B 83, 115323 (2011).

17. Bae, S. et al. Roll-to-roll production of 30-inch graphene films for transparent electrodes. Nature Nanotech. 5, 574-578 (2010).

18. Elias, D. C. et al. Control of Graphene's Properties by Reversible Hydrogenation: Evidence for Graphane. Science 323, 610-613 (2009).

19. Jaiswal, M. et al. Controlled Hydrogenation of Graphene Sheets and Nanoribbons. ACS Nano 5, 888-896 (2011).

20. Matis, B. R. et al. Surface Doping and Band Gap Tunability in Hydrogenated Graphene. ACS Nano 6, 17-22 (2012).

21. Marianer, S. \& Shklovskii, B. Effective temperature of hopping electrons in a strong electric field. Physical Review B 46, 13100-13103 (1992).

22. Nair, R. R. et al. Fluorographene: a two-dimensional counterpart of Teflon. Small 6, 2877-2884 (2010).

23. Ferrari, A. C. Raman spectroscopy of graphene and graphite: Disorder, electronphonon coupling, doping and nonadiabatic effects. Solid State Commun. 143, 47-57 (2007)

24. Lucchese, M. M. et al. Quantifying ion-induced defects and Raman relaxation length in graphene. Carbon 48, 1592-1597 (2010).

25. Martins Ferreira, E. H. et al. Evolution of the Raman spectra from single-, few-, and many-layer graphene with increasing disorder. Phys. Rev. B 82, 125429 (2010).

26. Ryu, S., Maultzsch, J., Han, M., Kim, P. \& Brus, L. E. Raman spectroscopy of lithographically patterned graphene nanoribbons. ACS Nano 5, 4123-4130 (2011).

27. Eckmann, A. et al. Probing the nature of defects in graphene by Raman spectroscopy. Nano Lett. 12, 3925-3930 (2012).

28. Saito, R., Hofmann, M., Dresselhaus, G., Jorio, A. \& Dresselhaus, M. S. Raman spectroscopy of graphene and carbon nanotubes. Adv. Phys. 60, 413-550 (2011).

29. Rappoport, T. G., Uchoa, B. \& Castro Neto, A. H. Magnetism and magnetotransport in disordered graphene. Phys. Rev. B 80, 245408 (2009).

30. Kim, S. et al. Size-dependence of Raman scattering from graphene quantum dots: Interplay between shape and thickness. Appl. Phys. Lett. 102, 053108 (2013).

31. Casiraghi, C. et al. Raman Spectroscopy of Graphene Edges. Nano Lett. 9, 1433-1441 (2009).

32. Shklovskii, B. I. \& Efros, A. L. Electronic Properties of Doped Semiconductors. (Springer-Verlag, Berlin, New York, 1984).

33. Liu, G. \& Soonpaa, H. Electric-field and temperature effects in a two-dimensional system with strong localization. Phys. Rev. B 48, 5682-5684 (1993).

34. Tran, T. et al. Sequential tunneling and inelastic cotunneling in nanoparticle arrays. Phys. Rev. B 78, 075437 (2008).

35. Tran, T. et al. Multiple Cotunneling in Large Quantum Dot Arrays. Phys. Rev. Lett 95, 076806 (2005).

36. Edwards, J. T. \& Thouless, D. J. Numerical studies of localization in disordered systems. J. Phys. C: Solid State Phys. 5, 807-820 (1972).

37. Ponomarenko, L. A. et al. Chaotic Dirac billiard in graphene quantum dots. Science 320, 356-358 (2008).

38. Aleiner, I. L. \& Glazman, L. I. Mesoscopic Fluctuations of Elastic Cotunneling. Phys. Rev. Lett. 77, 2057-2060 (1996).

39. Milliken, F. \& Ovadyahu, Z. Observation of conductance fluctuations in large $\mathrm{In}_{2} \mathrm{O}_{3-\mathrm{x}}$ films. Phys. Rev. Lett. 65, 911-914 (1990).

40. Cronenwett, S. M., Patel, S. R., Marcus, C. M., Campman, K. \& Gossard, A. C. Mesoscopic Fluctuations of Elastic Cotunneling in Coulomb Blockaded Quantum Dots. Phys. Rev. Lett. 79, 2312-2315 (1997).

41. Raikh, M. \& Ruzin, I. Size effect in the longitudinal hopping conduction of a narrow two-dimensional channel. Phys. Rev. B 42, 11203-11207 (1990).

42. Strelniker, Y., Havlin, S., Berkovits, R. \& Frydman, A. Resistance distribution in the hopping percolation model. Phys. Rev. E 72, 016121 (2005).

43. Beloborodov, I., Lopatin, A. \& Vinokur, V. Universal description of granular metals at low temperatures: Granular Fermi liquid. Phys. Rev. B 70, 205120 (2004). 
44. Nguyen, V. L., Spivak, B. Z. \& Shklovskii, B. I. Hopping-conductivity fluctuations in small samples. JETP Lett. 43, 44-47 (1986).

45. Zhao, H. L., Spivak, B. Z., Gelfand, M. P. \& Feng, S. Negative magnetoresistance in variable-range-hopping conduction. Phys. Rev. B 44, 10760-10767 (1991).

46. Strelniker, Y., Berkovits, R., Frydman, A. \& Havlin, S. Percolation transition in a two-dimensional system of Ni granular ferromagnets. Phys. Rev. E 69, 065105 (2004).

47. Somoza, A., Ortuño, M. \& Prior, J. Universal Distribution Functions in TwoDimensional Localized Systems. Phys. Rev. Lett. 99, 116602 (2007).

48. Prior, J., Somoza, A. \& Ortuño, M. Conductance fluctuations and singleparameter scaling in two-dimensional disordered systems. Phys. Rev. B 72 024206 (2005)

49. Choe, D. H. \& Chang, K. J. Effect of dimensionality on the localization behavior in hydrogenated graphene systems. Nano Lett. 12, 5175-5180 (2012).

50. Rakhmanov, A. L., Rozhkov, A. V., Sboychakov, A. O. \& Nori, F. Phase separation of hydrogen atoms adsorbed on graphene and the smoothness of the graphenegraphane interface. Phys. Rev. B 85, 035408 (2012).

51. Savini, G., Ferrari, A. C. \& Giustino, F. First-Principles Prediction of Doped Graphane as a High-Temperature Electron-Phonon Superconductor. Phys. Rev. Lett. 105, 037002 (2010).

52. Allain, A., Han, Z. \& Bouchiat, V. Electrical control of the superconducting-toinsulating transition in graphene-metal hybrids. Nature Mater. 11, 590-594 (2012)

53. Clerk, A. A., Devoret, M. H., Girvin, S. M., Marquardt, F. \& Schoelkopf, R. J. Introduction to quantum noise, measurement, and amplification. Rev. Mod. Phys. 82, 1155-1208 (2010).

54. Regan, W. et al. A direct transfer of layer-area graphene. Appl. Phys. Lett. 96 , $113102(2010)$

\section{Acknowledgments}

We thank A.H. Castro Neto, V. Pereira, M.A. Cazalilla and J. Martin for helpful discussions. This work was supported by the Singapore Millennium Foundation-NUS Research Horizons award (R-144-001-271-592; R-144-001-271-646), and NUS-YIA

(R144-000-283-101)

\section{Author contributions}

A.P. and M.J. designed and performed the experiments. A.P. and M.J. analyzed the data. A.P. wrote the paper. B.-H.H. and J.-H.A. prepared graphene by chemical vapor deposition. Y.W. and K.P.L. hydrogenated graphene. All authors discussed the data and reviewed the manuscript. B.Ö. devised and supervised the project.

\section{Additional information}

Supplementary information accompanies this paper at http://www.nature.com/ scientificreports

Competing financial interests: The authors declare no competing financial interests.

How to cite this article: Pachoud, A. et al. Multiple Virtual Tunneling of Dirac Fermions in Granular Graphene. Sci. Rep. 3, 3404; DOI:10.1038/srep03404 (2013).

This work is licensed under a Creative Commons Attribution-

NonCommercial-NoDerivs 3.0 Unported license. To view a copy of this license, visit http://creativecommons.org/licenses/by-nc-nd/3.0 